Kansas State University Libraries

New Prairie Press

\title{
LOGISTIC REGRESSION ANALYSIS TO DETERMINE FACTORS CONTRIBUTING TO SUMMER FEEDLOT DEATHS
}

J. Clausen

A. M. Parkhurst

T. L. Mader

Follow this and additional works at: https://newprairiepress.org/agstatconference

Part of the Agriculture Commons, and the Applied Statistics Commons

\section{c) (1) () $\Theta$}

This work is licensed under a Creative Commons Attribution-Noncommercial-No Derivative Works 4.0 License.

\section{Recommended Citation}

Clausen, J.; Parkhurst, A. M.; and Mader, T. L. (2011). "LOGISTIC REGRESSION ANALYSIS TO DETERMINE FACTORS CONTRIBUTING TO SUMMER FEEDLOT DEATHS," Conference on Applied Statistics in Agriculture. https://doi.org/10.4148/2475-7772.1046

This is brought to you for free and open access by the Conferences at New Prairie Press. It has been accepted for inclusion in Conference on Applied Statistics in Agriculture by an authorized administrator of New Prairie Press. For more information, please contact cads@k-state.edu. 


\title{
LOGISTIC REGRESSION ANALYSIS TO DETERMINE FACTORS CONTRIBUTING TO SUMMER FEEDLOT DEATHS
}

\author{
J. Clausen ${ }^{1}$, A. M. Parkhurst ${ }^{1}$, and T. L. Mader ${ }^{2}$ \\ ${ }^{1}$ Department of Statistics, University of Nebraska-Lincoln \\ ${ }^{2}$ Department of Animal Science, University of Nebraska-Lincoln
}

\begin{abstract}
Summer heat has already been identified as a major factor for cattle deaths in the feedlot. This study attempts to assess what other factors contribute to and/or influence cattle deaths. Identifying multiple factors that contribute to summer feedlot deaths could aid feedlot managers in implementation of mitigation strategies and minimize the loss of nearly finished cattle. Daily pen, cattle, and nutritional characteristics were recorded and included in this generalized linear mixed model analysis. Cattle data were obtained from cattle pens at a single location from July 1 , 2010 to July 31, 2010. Hourly weather data were acquired from this feed yard while solar radiation was received from a neighboring town. Rather than using multiple weather variables, a single comprehensive climate index that summarizes several weather variables is used to capture the apparent feel of the weather. After reviewing the data, a statistical model is developed and odds ratios are computed for statistical inference. According to these odds ratios, cattle fed on severe west slopes had significantly higher odds of death than other types of slopes. Analysis of feed intake indicates pens consuming 16pounds of feed per head or less during July $16-18$ have higher odds of death than other consumption levels.
\end{abstract}

\section{Introduction}

In the Midwest and Plain states, losses of confined cattle exceeded 2,000 head during each of seven separate heat waves, which occurred over the past 15 years. The heat waves of 1995, 1999, 2006, and 2009 were particularly severe with documented cattle losses in Midwestern states approaching 5,000 or more head each year (Mader, 2003; Mader et al. 2006; Mader and Gaughan, 2009). Economic losses from reduced cattle performance likely exceed those associated with cattle death losses (approximately $\$ 1,400$ per head) by 5- to 10-fold. In July 2010 another Midwestern cattle feedlot experienced substantial deaths during an adverse heat episode. To investigate possible contributors to death loss associated with hot weather conditions, pen, cattle, and nutritional characteristics are analyzed at a single location. Furthermore, both quantity and associated changes of daily feed intake are examined to assess effects of metabolic heat flux. Major interest is on the type of ration and dietary feed additive consumed.

\section{Materials and Methods \\ 2.1 Data Set}

Feedlot data were obtained from a Midwest cattle feedlot for the period from July 1, 2010 to July 31,2010 . Since the focus of this study was on the deadly heat wave that occurred between July 16 and July 20, the data set was reduced to the period July 9, 2010 to July 23, 2010. This feed yard contains a total of 188 pens of cattle, with 174 pens for the period of interest. Management at this feed yard maintained daily records for variables contained in their database.

Following are descriptions of variables, italicized, that appear in the feedlot data set. PenDailyLotNumber is a number used to keep track of groups of cattle owned by individuals. If 
groups of cattle are large, multiple pens in the feedlot may have the same PenDailyLotNumber. PenDailyPenNumber is a number assigned to a pen of cattle to identify the location of the pen at the feed yard. These numbers are subjectively assigned. PenDailyDate is the day when values for each variable are recorded. Each line in the data set represents an observation recorded on a particular pen on a given day.

Several adjustments to the original data set were needed for this analysis. Because some rations are only given to a few pens on a particular day, days are grouped into three periods in an attempt to increase the number of pens receiving those rations for these periods. Period is a categorical variable that indicates the period when the observation occurred. Heat1 is July $9-15$, Heat 2 is July $16-18$, and Heat 3 is July $19-23$. This division can be thought of as preheatwave, during heatwave, and post-heatwave periods.

Other adjustments required new variables to identify the number of cattle that died on a given day and cumulative deaths for each PenDailyLotNumber. Because feedlot personnel did not record most cattle deaths for July 16 - 17 until Sunday July 18, deaths recorded on July 18 were re-distributed to July 16,17 , and 18 . According to managers, an estimated $25 \%$ of the deaths occurred on July $16,50 \%$ on July 17 , and $25 \%$ on July 18 . Therefore, PenDailyDeadsDistributed is the number of cattle that died in a pen on a particular day after distributing July 18 deaths to July 16, 17, and 18. If July 16 or 17 had some deaths already recorded, the July 18 distributed deaths were added to these deaths. Then, PenDailyHeadCountDistributed is the number of live cattle in the pen for a given day, and PenDailyPenTotal is the total number of live and dead cattle in a pen for a particular day. Next, the proportion of dead cattle in a pen on a given day is ProportionDead which is PenDailyDeadsDistributed divided by PenDailyPenTotal. This is how the occurrences of deaths were identified.

Further variable definitions are as follows: PenDailySex is the gender of the animals in the pen with categories $\mathrm{H}$ (heifers), $\mathrm{S}$ (steers), and $\mathrm{M}$ (mixed - both heifers and steers). PenDailyAvgWeight is the estimated average weight of an animal in the pen computed by taking the total weight of all animals in the pen divided by the number of animals in the pen. Because the cattle are not weighed every day, these values are estimated, based on feed intake and historical feedlot performance records for the type of cattle being fed. PenFeedIntake records the total weight of feed given to cattle in a particular pen on a certain day. DMIPerHead is PenFeedIntake divided by PenDailyPenTotal to obtain an estimated amount of feed consumed by individual animals within a pen. DMIDailyChange is DMIPerHead today minus DMIPerHead received the previous day. Therefore, DMIDailyChange will be positive if cattle receive more feed today and negative if cattle receive less feed today in comparison to yesterday. If the amount of feed does not change, then DMIDailyChange will be zero. Because groups of cattle are occasionally moved from pen to pen or new groups enter the feed yard, DMIDailyChange will not be available until the second day for which cattle are in their new pen.

Since ration is the focus for this data analysis, the following variables are of interest. BmRation is the type of feed given to cattle in a pen. Nine different rations were distributed to pens throughout the middle of July. Rations 1,2, and 3 are roughage-based feed consisting of approximately $60 \%, 75 \%$, and $85 \%$ corn, respectively. Rations 4 and 14 are steer and heifer finishing rations, respectively. Ration 7 is a High-DGS (Distiller Grains) grower with energy 
content similar to Ration 2. Rations 41 and 42 are steer and heifer rations containing Betaagonists; while ration 47 is a steer ration containing other Beta-agonists. RationGroup is the grouping of certain rations. PenDailyAvgDOF is the number of days a group of cattle have been fed at the feed yard. The longer cattle are at the feed yard, the larger the PenDailyAvgDOF. NumberDaysOnRation and DaysOnAOrBRation record the number of days a pen receives a given ration or rations 41,42 , or 47 , respectively. NumberDaysOnRation is a quantitative variable while DaysOnAOrBRation is a categorical variable.

More pen characteristics are provided by these next variables. BunkLengthFt determines the amount of space available for cattle to eat their feed from bunks. PenDepthFt is the length of the pen from front to back. PenSqFt is the amount of area in the pen, recorded in squared feet. SqFtPerHead which gives the amount of area for each animal is defined as PenSqFt divided by PenDailyPenTotal. Slope indicates the direction the pen is sloping. Flat, moderate east (ME), moderate northeast (MNE), moderate northwest (MNW), moderate west (MW), north (Nor), severe west (SevW), and south (Sou) are the possible slope directions at this feed yard. WaterTanks is the number of both fixed water tanks in the pen and galvanized water tanks added to the pen during the heat period. There are water tank combinations appearing in this data set: One shared and zero galvanized (1S0G), one not shared and zero galvanized (1NS0G), one shared and one galvanized (1S1G), and one not shared and one galvanized (1NS1G).

Lastly, since many of the quantitative variables reflect transitions of cattle among pens, nearly all quantitative variables are converted into new categorical variables for ease of interpretation. PenDailyAvgWeightCateg, DMIPerHeadCateg, DMIDailyChangeCateg, PenDailyAvgDOFCateg, BunkLengthFtCateg, PenDepthFtCateg, and SqFtPerHeadCateg are all four level category variables formed from quartiles. For simplicity, midpoints of quartiles will be used when referring to quartiles.

Hourly weather data were available at this Midwest cattle feedlot from July 9 - July 23 due to availability of equipment used by other researchers. All weather variables except solar radiation were obtained at the feed yard. Solar radiation values were obtained from a neighboring town approximately 4 miles away. Since the feedlot data have only one entry for each day, the median of the 24 hourly values of a comprehensive climate index was used to create one daily value. The comprehensive climate index developed by Mader et al. (2010) uses ambient temperature, relative humidity, wind speed, and solar radiation to provide a measure to capture the conditions of the day. Since this index contains multiple weather measures, Median CCIV3.2, $\left({ }^{\circ} \mathrm{F}\right)$ is the one weather variable that appears in this analysis and is always a quantitative variable. It provides a relative indicator of the environmental conditions surrounding an animal. It mathematically quantifies how RAD, WS, and RH interact with ambient temperature $(\mathrm{Ta})$ to produce an apparent temperature. The CCI was developed under environmental conditions associated with Ta from approximately -30 to $45^{\circ} \mathrm{C}$ and provides an adjustment to Ta for relative humidity, wind speed, and radiation. (Mader, 2010) Lagged Median CCI is the previous day's Median CCIv3.2. Yesterday's Median CCI is considered because this value might be associated with the current values for many of the variables used to describe the data.

\subsection{Statistical Methods}

Data analysis is implemented using the Statistical Analysis System (SAS). Descriptive statistics are obtained using proc freq, proc means, and proc gplot SAS procedures to summarize and plot 
collected feed yard data. Within SAS, the proc glimmix procedure is used to construct a statistical model for proportions data. The model has a Linear Predictor,

$$
\eta_{\mathrm{ijklmnrst}}=\eta+\alpha_{\mathrm{i}}+\beta_{\mathrm{j}}+\gamma_{\mathrm{k}}+\delta_{1}+\rho_{\mathrm{m}}+\tau_{\mathrm{n}}+\phi_{\mathrm{r}}+\mathrm{d}_{\mathrm{s}}+\theta \mathrm{X}_{\mathrm{t}(\mathrm{i})}+(\alpha \rho)_{\mathrm{im}}+(\alpha \tau)_{\mathrm{in}}+(\delta \phi)_{\mathrm{lr}}
$$

$\mathrm{i}=$ Heat 1, Heat 2 , Heat 3

$\mathrm{j}=4,14,12-37,41,42-47$

$\mathrm{k}=$ Flat, ME, MNE, MNW, MW, Nor, SevW, Sou

$1=\mathrm{Q} 1, \mathrm{Q} 2, \mathrm{Q} 3, \mathrm{Q} 4$

$\mathrm{m}=\mathrm{Q} 1, \mathrm{Q} 2, \mathrm{Q} 3, \mathrm{Q} 4$

$\mathrm{n}=\mathrm{Q} 1, \mathrm{Q} 2, \mathrm{Q} 3, \mathrm{Q} 4$

$\mathrm{r}=\mathrm{Q} 1, \mathrm{Q} 2, \mathrm{Q} 3, \mathrm{Q} 4$

$\mathrm{s}=1,2,3,4, \ldots, \mathrm{p}$ where $\mathrm{p}$ is the number of pens at level $\mathrm{i}, \mathrm{j}$,

$\mathrm{k}, 1, \mathrm{~m}, \mathrm{n}, \mathrm{r}$

$\mathrm{t}=83.81,83.61,88.62,83.47,87.92,89.4,86.19,93.47,92.2, \quad(\mathrm{X}=$ Median $\mathrm{CCI})$

$92.38,86.14,82.19,85.02,85.37,85.23$

Response Variable:

Distributional Assumption:

$$
\mathrm{y}_{\mathrm{ijklmnrst}} \mid \mathrm{d}_{\mathrm{s}} \sim \operatorname{Binomial}\left(\mathrm{N}_{\mathrm{ijklmnrst}}, \pi_{\mathrm{ijklmnrst}}\right)
$$

Link:

$$
\eta_{\mathrm{ijklmnrst}}=\log \left(\frac{\pi_{\mathrm{ijklmnrst}}}{1-\pi_{\mathrm{ijklmnrst}}}\right)=\operatorname{logit}\left(\pi_{\mathrm{ijklmnrst}}\right)
$$

$y_{i j k l m n r s t}$ is number of cattle deaths during $\mathrm{i}^{\text {th }}$ period, receiving $\mathrm{j}^{\text {th }}$ ration, on $\mathrm{k}^{\text {th }}$ slope, in $\mathrm{l}^{\text {th }}$ pen average weight quartile, $\mathrm{m}^{\text {th }}$ dry matter intake quartile, $\mathrm{n}^{\text {th }}$ dry matter intake change quartile, $\mathrm{r}^{\text {th }}$ square feet per head quartile for $\mathrm{s}^{\text {th }}$ pen, and $\mathrm{t}^{\text {th }}{ }^{\text {median }} \mathrm{CCI}$.

$N_{i j k l m n r s t}$ is number of cattle during $\mathrm{i}^{\text {th }}$ period, receiving $\mathrm{j}^{\text {th }}$ ration, on $\mathrm{k}^{\text {th }}$ slope, in $\mathrm{1}^{\text {th }}$ pen average weight quartile, $m^{\text {th }}$ dry matter intake quartile, $n^{\text {th }}$ dry matter intake change quartile, $\mathrm{r}^{\text {th }}$ square feet per head quartile for $\mathrm{s}^{\text {th }}$ pen, and $\mathrm{t}^{\text {th }}{ }^{\text {median }} \mathrm{CCI}$.

$\pi_{i j k l m n r s t}$ is population probability of death during $\mathrm{i}^{\text {th }}$ period, receiving $\mathrm{j}^{\text {th }}$ ration, on $\mathrm{k}^{\text {th }}$ slope, in $\mathrm{l}^{\text {th }}$ pen average weight quartile, $\mathrm{m}^{\text {th }}$ dry matter intake quartile, $\mathrm{n}^{\text {th }}$ dry matter intake change quartile, $\mathrm{r}^{\text {th }}$ square feet per head quartile for $\mathrm{s}^{\text {th }}$ pen, and $\mathrm{t}^{\text {th }}{ }^{\text {median }} \mathrm{CCI}$.

$\sigma_{d}^{2}$ is the population variance for the $s^{\text {th }}$ pen

$\eta$ is the overall mean

$\alpha_{i}$ is the fixed effect of the ith level of period

$\beta_{\mathrm{j}}$ is the fixed effect of the $\mathrm{jth}$ level of ration group

$\gamma_{\mathrm{k}}$ is the fixed effect of the kth level of slope

$\delta_{1}$ is the fixed effect of the lth pen average weight quartile

$\rho_{\mathrm{m}}$ is the fixed effect of the mth dry matter intake per head quartile

$\tau_{\mathrm{n}}$ is the fixed effect of the $\mathrm{nth}$ dry matter intake change quartile

$\phi_{\mathrm{r}}$ is the fixed effect of the rth square feet per head quartile

$d_{s}$ is the random effect of the sth pen 
$\theta$ is the slope coefficient for median CCI

$\mathrm{X}_{\mathrm{t}(\mathrm{i})}$ is the $\mathrm{t}$-th level of median CCI within the ith period, a direct variable

$(\alpha \rho)_{\text {im }}$ is the fixed interaction effect of the ith level of period and mth dry matter intake per head quartile

$(\alpha \tau)_{\text {in }}$ is the fixed interaction effect of the ith level of period and nth dry matter intake change quartile

$(\delta \phi)_{\mathrm{Ir}}$ is the fixed interaction effect of the lth pen average weight quartile and the rth square feet per head quartile

Since the feedlot data do not have a repeated measure experimental design, proc glimmix was not executed in a fashion consistent with a repeated measures study. However, several proc glimmix options are added to assist in the analysis of the data. A Laplace integral approximation procedure is implemented in order for a real likelihood function to be available for likelihood ratio tests and information criteria. Another reason for the Laplace approximation procedure is proc glimmix has difficulty converging without this option. In addition, the Morel et al. (2003) bias correction to the classical sandwich estimator is used to help control the bias in standard errors. If standard errors are underestimated, then F-statistics are overestimated, and the Type I error rate increases.

The first step in the model building procedure was forward selection with significance level for a variable to enter (sle) the model $\leq 0.20$. The higher significance level is used to control the Type II error because not including an important variable is much worse than including an unimportant variable. Then, backwards elimination was performed with the significance to stay set to $\leq 0.20$. For the final model, interaction terms were with added with sle $\leq 0.20$.

The fit statistics, -2 log likelihood and information criteria, provide other method ways to compare competing models. Since the Laplace integral approximation method results in using a real likelihood function, these information criteria can be used for model comparisons. A model with smaller information criteria is preferable. Furthermore, the Pearson Chi-Square/DF is a valid over-dispersion statistic for the conditional distribution of count data. If this value is near 1 , then over-dispersion is not considered a problem for the model. Because the data are not being modeled as counts, but rather as proportions, the Pearson Chi-Square/DF does not offer formal insight into the appropriateness of the model.

\section{Results and Discussion}

\subsection{Descriptive Statistics}

In order to understand the structure of this survey data, descriptive statistics and frequency table with non-zero pen counts are analyzed to ensure that information is available for certain levels of variables. 174 pens from the feed yard are included in this analysis and counted each day from July 9 - July 23 . However, data from some pens were not recorded on certain days; therefore, the total number of pens is 2475 . Thus, total pen count may change due to missing data for various variables.

Since each day has at least 160 pens, sufficient information is available to observe the death counts on each day included in the analysis. Before the heat wave, few deaths occurred. Then, as 
weather conditions became hotter, deaths rose dramatically. Finally, deaths began to decline. Thus, days are combined into three periods: Pre-wave Heat1 from July 9 - July 15 (9 deaths); Heat2 from July 16 - July 18 (639 deaths); and Post-waveHeat3 from July 19 - July 23 (40 deaths).

Each ration has at least 29 pens. Rations 1, 2, 3, 7, 41, 42, and 47 have 5 or less deaths. Because these death counts are extremely small, grouping rations is needed in order to improve the validity of inference. Rations 1, 2, 3, and 7 are grouped together because of similar roughedbased feed characteristics ( 8 deaths); rations 41, 42, and 47 form a Beta-agonist group with 12 deaths; and rations 4 and 14 remain in their own groups because of abundant death counts of 175 and 493, respectively.

Each slope has information since pen frequencies are greater than 100 . Pens with flat slopes appear most frequently $(\mathrm{n}=826)$. Flat and moderate west have the largest number of deaths (over $100)$. Severe west and moderate east have near 100. Moderate northeast and south have number of death around 50, while north and moderate northwest have the smallest number of deaths ( $\leq$ 20).

Most deaths occurred when PenDailyAvgWeight was in second and third quartiles. Lighter cattle with an average weight about $670 \mathrm{lbs}$ had the least number of deaths, and heavier cattle with an average weight near $1240 \mathrm{lbs}$ had the second smallest death count. This feed yard contained far more heifers than steers or pens with both heifers and steers. Consequently, heifers had the greatest number of deaths while steers and mixed pens had many fewer deaths. Accounting for PenDailyAvgWeight, heifers had large death counts in all weight quartiles while steers and mixed pens tend to have deaths in the upper weight quartiles. When analyzing deaths, gender is confounded with weight.

Since each day and ration combination contains at least one pen, we can summarize the daily deaths for each ration. Most days have no death or a small number of deaths ( $1 \sim 2$ deaths), except days 07/16, 07/17, 07/18 (136 deaths, 370 deaths, 133 deaths, respectively). When constructing a statistical model for these data, days are grouped into periods in order to eliminate zero deaths. Even if rations 1, 2, 3, and 7 were already grouped together, their death count would still be zero for many days in July. Therefore, both rations and days must be grouped to eliminate small cell counts.

Ration and PenDailyAvgWeight combinations offer an example when the pen counts are occasionally zero, Table 1 . The data set contains pens for those Ration and PenDailyAvgWeight combinations and the death counts that have statistical meaning. Consequently, non-highlighted entries indicate no pen data are present in the data set for the particular ration and pen average weight combination, and of course, no deaths occurred. Observing the highlighted cells, one should notice that rations 1,2 , and 7 are fed to lighter cattle while rations 41,42 , and 47 are fed to heavier cattle. Only rations 3,4 , and 14 are fed to cattle in all weight categories. Confounding such as this inhibits the ability to analyze interactions between certain variables because sufficient information is not available for all factor level combinations. 
Table 1: Frequency of Ration by PenDailyAvgWeightCateg Death Counts

\begin{tabular}{|l|l|l|l|l|l|}
\hline \multirow{2}{*}{ Ration } & \multicolumn{4}{|c|}{ PenDailyAvgWeightCateg } & \multirow{2}{*}{ Total } \\
\cline { 2 - 5 } & Q1 & Q2 & Q3 & Q4 & \\
\hline 1 & 2 & 0 & 0 & 0 & 2 \\
\hline 2 & 0 & 0 & 0 & 0 & 0 \\
\hline 3 & 4 & 0 & 0 & 0 & 4 \\
\hline 4 & 1 & 46 & 53 & 75 & 175 \\
\hline 7 & 2 & 0 & 0 & 0 & 2 \\
\hline 14 & 36 & 171 & 187 & 99 & 493 \\
\hline 41 & 0 & 0 & 0 & 5 & 5 \\
\hline 42 & 0 & 0 & 1 & 1 & 2 \\
\hline 47 & 0 & 0 & 0 & 5 & 5 \\
\hline Total & 45 & 217 & 241 & 185 & 688 \\
\hline
\end{tabular}

After rations and days are combined into ration groups, the death counts are obtained. Rations 4 and 14 during Heat2 (heat) result in the greatest number of deaths. Small death counts continue to be a problem for certain combinations. Seven out of 12 ration and period interactions contain 4 deaths or less. Due to these sparse death counts, analyzing ration group and period interaction is not considered. Even though interaction counts are sparse, grouping rations and grouping days will allow ration group and period to have large enough death counts for inclusion in building a statistical model. Lastly, pen counts are non-zero for each ration group and period combination.

In addition to frequency table, graphs can provide insight for understanding conditions throughout the two-week period. As noted earlier, weather conditions became extremely hot during July 16 - July 18 . A plot of the Median CCI of the 24 hourly CCI readings vs. Day shows spikes in Median CCI to about $88 \mathrm{~F}$ and $89 \mathrm{~F}$ on July 11, July 13, and July 14 with a weak cooling day on July 12, Figure 1. A mean daily CCI of $88 \mathrm{~F}$ or above is severe and could potentially lead to cattle deaths (Mader, 2011). From July 14, Median CCI declines slightly on July 15 and abruptly spikes on July 16 to approximately $93 \mathrm{~F}$. At this point, cattle have already experienced high CCI values for several days. But now, cattle begin to encounter severe CCI values over a three-day period. Prolonged exposure to these CCI values helped contribute to many deaths at the feed yard, as shown in Table 2. After July 18, Median CCI values rapidly decline the next two days while returning to regular summer conditions within the next three days. Even though the Median CCI declined on July 19, 30 cattle died after surviving the worst weather conditions.

Figure 1: Median CCIv3.2, $\left(\mathrm{F}^{\circ}\right)$, for Each Day

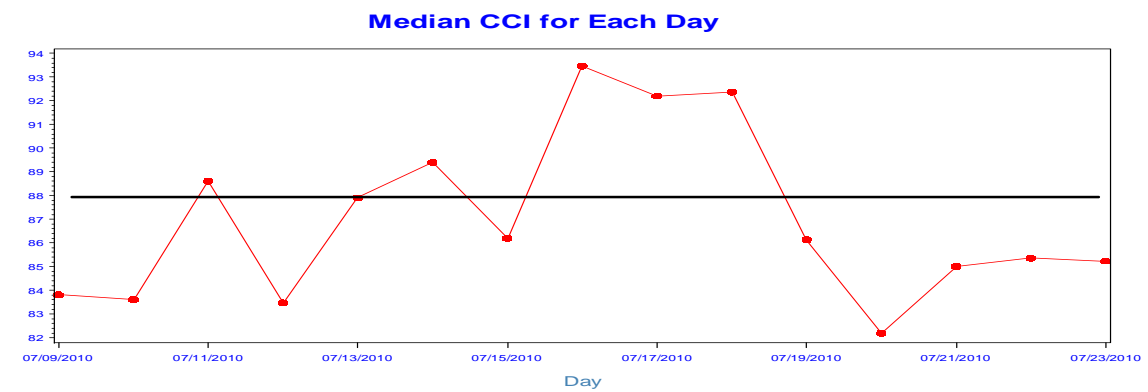


Change in the amount of feed consumed by cattle from one day to the next is a way to monitor the body energy level during intense summer weather conditions. According to feed yard protocol, operators do not purposely restrict the amount of feed consumed by cattle at the feed yard. If some feed remained in the bunks at feeding time, then the operators would reduce the amount of feed given to the pen. Figure 2 shows median change in dry matter intake for pens consuming a particular ration on a specific day. Median change in matter intake fluctuates around zero from July 9 - July 15 . Then, July 16 shows slight declines in median dry matter intake for rations 3, 4, 14, 41, 42, and 47. After experiencing a median CCI around $93 \mathrm{~F}$ on July 16 , July 17 indicates cattle are reaching their maximum energy consumption capacity, and the change in dry matter intake decreases dramatically for rations 4, 14, 41, 42, and 47. After July 17 , these cattle regain their appetite and compensate in feed intake even though median CCI values are still high (Mader, 2011). Note that when median CCI troughed at $82 \mathrm{~F}$ on July 20, all rations had a positive change in median dry matter intake.

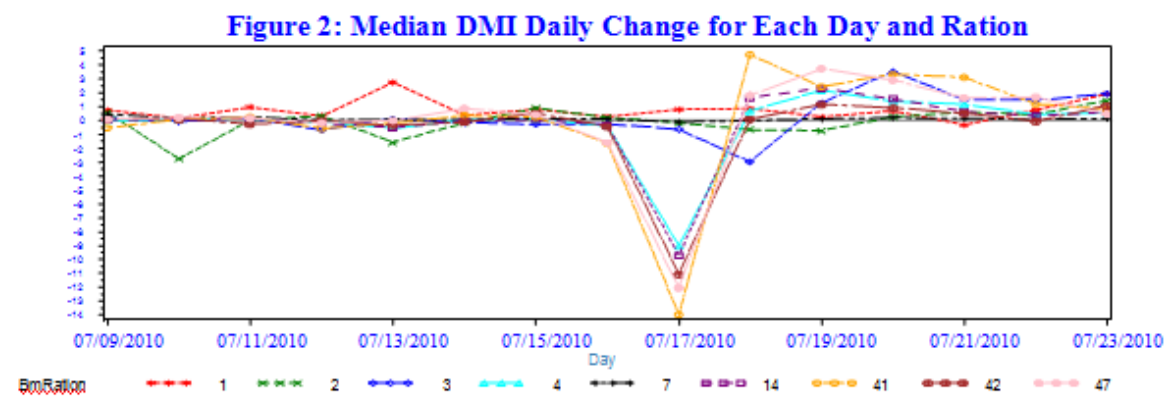

Figure 3 summarizes daily activity at the feed yard. The average of proportions computed for each pen receiving a particular ration on a certain day is used to observe days when the proportion of deaths abruptly changes. For July 9 - July 15, average proportion dead remains near zero. Overall, as median CCI increases for July 16 - July 18, average proportion dead increases during July $16-17$. Then, as median CCI tends to decrease for July $18-23$, the average proportion dead declines as well. Hence, average proportion dead tends to oscillate in conjunction with median CCI. Of the rations, ration 41 has two large peaks on July 17 and 19. Some cattle on ration 41 died after the first day of median CCI values in the 90 F's while others died after the third day of high CCI values.

Figure 3: Average Proportion Dead for Each Day and Ration

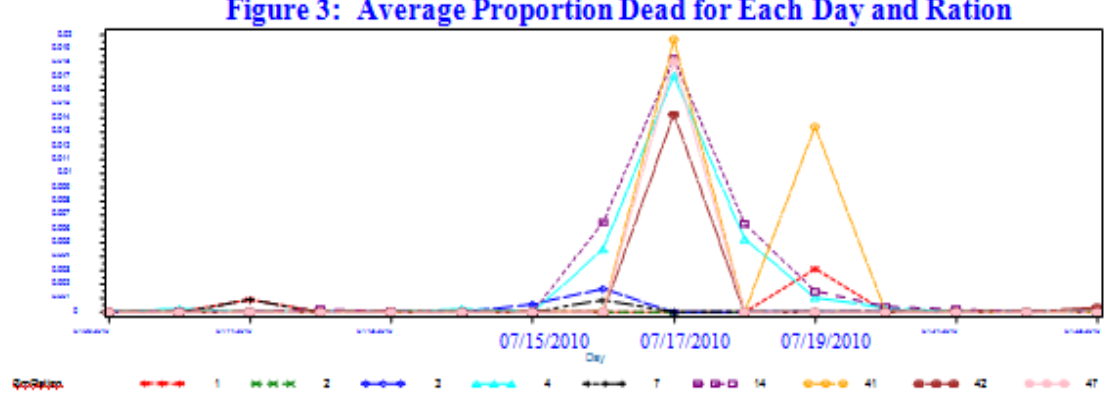

Finally, the average proportion dead for each gender and pen average weight quartile is summarized in Figure 4. As for the mixed pens containing both heifers and steers, the average proportion dead remains at zero until Q4 $(1240 \mathrm{lbs})$ is reached which results in the highest 
average proportion dead in the plot. Next, steers show a large spike in average proportion dead at Q2 (900 lbs) of pen average weight and then slightly declines for Q3 (1040 lbs) while increasing moderately at Q4 (1240 lbs). Lastly, heifers show a steady incline in the average proportion dead for the first three quartiles (670, 900, and $1040 \mathrm{lbs})$ and moderately declines for Q4 (1240 lbs). Overall, all three gender categories indicate an increase in the average proportion dead as pen average weight increases.

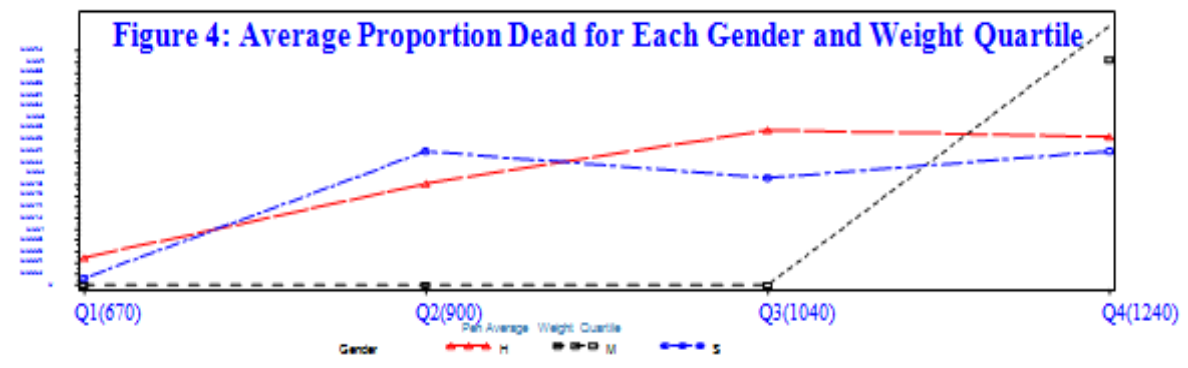

\subsection{Statistical Model Results}

Overall fit of the final model and a summary are provided in Table 2. The Type III Tests of Fixed Effects output displays the significance of each term in the final model. Even though DMI Daily Change Category has a p-value much greater than 0.20, this main effect remains in the model because this term is involved in a significant two-way interaction with Period. Because Ration Group and Slope are not involved in interactions, these terms will be analyzed as main effects. As for the other terms involved in interactions, only those interactions will be analyzed and not their main effects.

Table 2: Model Summary for the fixed effects from proc glimmix

\begin{tabular}{l|c} 
Effects & P-value \\
\hline Period & 0.0009 \\
Ration_Group & 0.1741 \\
Slope & 0.0695 \\
PenDailyAvgWeightCateg & $<.0001$ \\
DMI_per_headCateg & $<.0001$ \\
DMI_Daily_ChangeCateg & 0.7767 \\
sq_ft_per_headCateg & 0.0297 \\
Median_CCIv3_2_F & 0.1468 \\
Period*DMI_per_headCateg & 0.0261 \\
Period*DMI_Daily_ChangeCateg & 0.0992 \\
PenDailyAvgWeightCateg*sq_ft_per_headCateg & 0.0480
\end{tabular}

Simple effects of one factor at specific levels of the other factor are shown in Table 3 A. Conditioning on level of DMIPerHeadCateg quartiles, the estimated odds of death for one period are compared with another period. Only four comparisons show significant differences in the odds of death. When cattle fall into Q2 $(18 \mathrm{lbs})$ of DMIPerHeadCateg, the odds of death for Heat2 (heat) are significantly larger than Heat3 (post-heat) (Lower Odds Ratio $=1.256>1$ ). When cattle are in Q3 $(21 \mathrm{lbs})$ of DMIPerHeadCateg, Heat2 has a larger odds of death ratio than both Heat1 (pre-heat) (Heat1 vs. Heat2 Upper Odds Ratio $=0.219<1$ ) and Heat3 (Heat2 vs. 
Heat3 Lower Odds Ratio $=1.058>1)$. Lastly, Q4 $(28 \mathrm{lbs})$ of DMIPerHeadCateg shows significantly lower odds of death for Heat1 than Heat2 (Upper Odds Ratio $=0.224<1$ ). For the three dry matter intake quartiles discussed, Heat 2 shows a trend for larger odds of death.

Conditioning on Period, contrasts between quartiles of DMIPerHeadCateg are shown in Table 3 B. During Heat1, the odds of death are higher for Q1 $(10 \mathrm{lbs})$ of DMIPerHeadCateg than Q3 and Q4. For Heat2, the odds of death are higher for DMIPerHeadCateg Q1 when compared individually with Q2, Q3, and Q4. And for the last period, only one contrast is significant. During Heat3, the odds of death are higher for Q1 of DMIPerHeadCateg than Q2. A common theme among all periods is cattle consuming less feed tend to have a larger odds ratio of death. This suggests that cattle that are dying have already reduced their intake in an effort to lower metabolic heat load but for some cattle not significantly enough to prevent death.

Table 3: Odds Ratios for Period by DMIPerHeadCateg Quartiles interactions A

\begin{tabular}{|l|l|l|l|l|l|}
\hline DMIPerHeadCateg & Period & Period & Odds Ratio & $\begin{array}{l}\text { Bonferroni Adj } \\
\text { Lower Odds Ratio }\end{array}$ & $\begin{array}{l}\text { Bonferroni Adj } \\
\text { Upper Odds Ratio }\end{array}$ \\
\hline \multirow{4}{*}{ Q1 } & Heat 1 & Heat 2 & 0.401 & 0.023 & 6.930 \\
\cline { 2 - 7 } & Heat 1 & Heat 3 & 2.236 & 0.147 & 33.961 \\
\cline { 2 - 7 } & Heat 2 & Heat 3 & 5.574 & 0.758 & 40.990 \\
\hline \multirow{4}{*}{ Q2 } & Heat 1 & Heat 2 & 0.088 & 0.005 & 1.570 \\
\cline { 2 - 7 } & Heat 1 & Heat 3 & 0.969 & 0.063 & 15.031 \\
\cline { 2 - 7 } & Heat 2 & Heat 3 & 11.045 & 1.256 & 97.169 \\
\hline Q3 & Heat 1 & Heat 2 & 0.019 & 0.002 & 0.219 \\
\cline { 2 - 7 } & Heat 1 & Heat 3 & 1.007 & 0.019 & 54.266 \\
\cline { 2 - 6 } & Heat 2 & Heat 3 & 51.629 & 1.058 & $>999.999$ \\
\hline Q4 & Heat 1 & Heat 2 & 0.029 & 0.004 & 0.224 \\
\cline { 2 - 6 } & Heat 1 & Heat 3 & 1.536 & 0.010 & 238.850 \\
\cline { 2 - 6 } & Heat 2 & Heat 3 & 53.579 & 0.320 & \\
\hline
\end{tabular}

B

\begin{tabular}{|c|c|c|c|c|c|}
\hline Period & $\begin{array}{l}\text { DMIPerHead } \\
\text { Categ }\end{array}$ & $\begin{array}{l}\text { DMIPerHead } \\
\text { Categ }\end{array}$ & Odds Ratio & $\begin{array}{l}\text { Bonferroni Adj Lower } \\
\text { Odds Ratio }\end{array}$ & $\begin{array}{l}\text { Bonferroni Adj Upper } \\
\text { Odds Ratio }\end{array}$ \\
\hline \multirow[t]{6}{*}{ Heat 1} & Q1 & Q2 & 11.579 & 0.253 & 530.222 \\
\hline & Q1 & Q3 & 56.046 & 1.764 & $>999.999$ \\
\hline & Q1 & $\mathrm{Q} 4$ & 47.286 & 2.120 & $>999.999$ \\
\hline & $\mathrm{Q} 2$ & Q3 & 4.840 & 0.166 & 141.521 \\
\hline & $\mathrm{Q} 2$ & $\mathrm{Q} 4$ & 4.084 & 0.208 & 80.230 \\
\hline & $\mathrm{Q} 3$ & $\mathrm{Q} 4$ & 0.844 & 0.050 & 14.160 \\
\hline \multirow[t]{6}{*}{ Heat 2} & Q1 & $\mathrm{Q} 2$ & 2.533 & 1.453 & 4.416 \\
\hline & Q1 & Q3 & 2.724 & 1.739 & 4.265 \\
\hline & Q1 & Q4 & 3.379 & 2.046 & 5.580 \\
\hline & $\mathrm{Q} 2$ & Q3 & 1.075 & 0.601 & 1.925 \\
\hline & $\mathrm{Q} 2$ & $\mathrm{Q} 4$ & 1.334 & 0.697 & 2.553 \\
\hline & $\mathrm{Q} 3$ & $\mathrm{Q} 4$ & 1.240 & 0.731 & 2.104 \\
\hline \multirow[t]{6}{*}{ Heat 3} & Q1 & $\mathrm{Q} 2$ & 5.018 & 1.554 & 16.210 \\
\hline & Q1 & Q3 & 25.227 & 0.873 & 728.964 \\
\hline & Q1 & Q4 & 32.476 & 0.185 & $>999.999$ \\
\hline & $\mathrm{Q} 2$ & Q3 & 5.027 & 0.130 & 194.857 \\
\hline & $\mathrm{Q} 2$ & Q4 & 6.471 & 0.035 & $>999.999$ \\
\hline & Q3 & $\mathrm{Q} 4$ & 1.287 & 0.003 & 623.749 \\
\hline
\end{tabular}


Next, the results for the interaction between Period and DMIDailyChange quartiles are explored in Table $4 \mathrm{~A}$. When DMIDailyChange is in Q1 $(-11 \mathrm{lbs})$, the odds of death are significantly higher for Heat2 than Heat1 (Heat1 vs. Heat2 Upper Odds Ratio $=0.253<1$ ) and Heat3 (Heat2 vs. Heat 3 Lower Odds Ratio $=3.471>1$ ). DMIDailyChange values in Q1 correspond to decreases in feed intake. Figure 2 shows a sharp decline in median dry matter intake during a large median CCI value on July 17 within period Heat2. Hence, these results are an indication that hot weather conditions during July $16-18$ are associated with the odds of death. Similarly, DMIDailyChange in Q2 (-0.10 lbs) shows a larger odds of death ratio for Heat2 than Heat1. Once again, Heat 2 stands out. When comparisons are made within period (Table 4 B), only two contrasts for DMIDailyChange are significant. During Heat2, the odds of death are higher for Q3 and Q4 (0.35 and $7 \mathrm{lbs})$ of dry matter intake change than Q1. Q3 and Q4 correspond to increases in feed intake. This result suggests cattle consuming larger quantities of feed during Heat 2 are less affected by the heat stress and have larger odds of surviving the harsh weather conditions.

Table 4: Odds Ratios for Period by DMIDailyChange Quartiles interactions

A

\begin{tabular}{|l|l|l|l|l|l|}
\hline $\begin{array}{l}\text { DMIDaily } \\
\text { Change }\end{array}$ & Period & Period & Odds Ratio & $\begin{array}{l}\text { Bonferroni Adj } \\
\text { Lower Odds Ratio }\end{array}$ & $\begin{array}{l}\text { Bonferroni Adj } \\
\text { Upper Odds Ratio }\end{array}$ \\
\hline Q1 & Heat 1 & Heat 2 & 0.016 & 0.023 & 6.930 \\
\cline { 2 - 6 } & Heat 1 & Heat 3 & 1.927 & 0.037 & 100.990 \\
\cline { 2 - 6 } & Heat 2 & Heat 3 & 119.339 & 3.471 & $>999.999$ \\
\hline \multirow{4}{*}{ Q2 } & Heat 1 & Heat 2 & 0.087 & 0.012 & 0.642 \\
\cline { 2 - 6 } & Heat 1 & Heat 3 & 2.063 & 0.092 & 46.161 \\
\cline { 2 - 6 } & Heat 2 & Heat 3 & 23.855 & 0.879 & 647.736 \\
\hline Q3 & Heat 1 & Heat 2 & 0.126 & 0.010 & 1.607 \\
\cline { 2 - 6 } & Heat 1 & Heat 3 & 1.205 & 0.085 & 17.132 \\
\cline { 2 - 6 } & Heat 2 & Heat 3 & 9.592 & 0.619 & 148.635 \\
\hline \multirow{4}{*}{ Q4 } & Heat 1 & Heat 2 & 0.112 & 0.003 & 4.404 \\
\cline { 2 - 6 } & Heat 1 & Heat 3 & 0.699 & 0.014 & 33.851 \\
\cline { 2 - 6 } & Heat 2 & Heat 3 & 6.237 & 0.537 & 72.491 \\
\hline
\end{tabular}

$\mathrm{B}$

\begin{tabular}{|c|c|c|c|c|c|}
\hline Period & $\begin{array}{l}\text { DMIDaily } \\
\text { Change }\end{array}$ & $\begin{array}{l}\text { DMIDaily } \\
\text { Change }\end{array}$ & Odds Ratio & $\begin{array}{lll}\text { Bonferroni } & \text { Adj } & \text { Lower } \\
\text { Odds Ratio } & & \\
\end{array}$ & $\begin{array}{lll}\text { Bonferroni } & \text { Adj } & \text { Upper } \\
\text { Odds Ratio } & & \end{array}$ \\
\hline \multirow[t]{6}{*}{ Heat 1} & Q1 & Q2 & 0.035 & 0.015 & 8.045 \\
\hline & Q1 & Q3 & 0.362 & 0.012 & 11.031 \\
\hline & Q1 & Q4 & 0.355 & 0.003 & 41.665 \\
\hline & $\mathrm{Q} 2$ & $\mathrm{Q} 3$ & 1.034 & 0.096 & 11.197 \\
\hline & Q2 & $\mathrm{Q} 4$ & 1.012 & 0.018 & 56.505 \\
\hline & Q3 & $\mathrm{Q} 4$ & 0.979 & 0.012 & 77.761 \\
\hline \multirow{6}{*}{ Heat 2} & Q1 & $\mathrm{Q} 2$ & 1.877 & 0.956 & 3.684 \\
\hline & Q1 & Q3 & 2.820 & 1.106 & 7.190 \\
\hline & Q1 & $\mathrm{Q} 4$ & 2.463 & 1.835 & 3.305 \\
\hline & $\mathrm{Q} 2$ & Q3 & 1.502 & 0.474 & 4.759 \\
\hline & $\mathrm{Q} 2$ & Q4 & 1.312 & 0.657 & 2.618 \\
\hline & Q3 & Q4 & 0.873 & 0.330 & 2.311 \\
\hline \multirow[t]{6}{*}{ Heat 3} & Q1 & Q2 & 0.375 & 0.007 & 19.919 \\
\hline & Q1 & Q3 & 0.227 & 0.008 & 6.464 \\
\hline & Q1 & Q4 & 0.129 & 0.007 & 2.531 \\
\hline & $\mathrm{Q} 2$ & Q3 & 0.604 & 0.028 & 13.257 \\
\hline & Q2 & Q4 & 0.343 & 0.021 & 5.505 \\
\hline & $\mathrm{Q} 3$ & $\mathrm{Q} 4$ & 0.568 & 0.103 & 3.138 \\
\hline
\end{tabular}


Lastly, the interaction between PenDailyAvgWeight quartiles and SqFtPerHead quartiles are presented in Table 5. When SqFtPerHead is in Q2 $\left(190 \mathrm{ft}^{2}\right)$, the odds of death are significantly higher for quartiles of PenDailyAvgWeight Q2, 3, and 4 (900, 1040, and $1240 \mathrm{lbs}$ ) than Q1 (670 lbs). For Q3 (240 $\left.\mathrm{ft}^{2}\right)$ of SqFtPerHead, the odds of death are higher for quartiles of pen average weight Q3 and 4 than Q1. Finally for SqFtPerHead in Q4 $\left(1350 \mathrm{ft}^{2}\right)$, the odds of death for PenDailyAvgWeight of Q4 is higher than Q1. A common observation among the square feet areas considered, heavier cattle have larger odds of death than lighter cattle. Hence, heavier cattle seem more sensitive to higher energy levels than lighter cattle across the pen densities.

Table 5: Odds Ratios for PenDailyAvgWeight quartiles by SqFtPerHead quartiles interactions

\begin{tabular}{|c|c|c|c|c|c|}
\hline SqFtPerHead & $\begin{array}{l}\text { PenDaily } \\
\text { AvgWeight }\end{array}$ & $\begin{array}{l}\text { PenDaily } \\
\text { AvgWeight }\end{array}$ & Odds Ratio & $\begin{array}{l}\text { Bonferroni Adj Lower } \\
\text { Odds Ratio }\end{array}$ & $\begin{array}{l}\text { Bonferroni Adj Upper } \\
\text { Odds Ratio }\end{array}$ \\
\hline \multirow[t]{6}{*}{ Q1 } & Q1 & Q2 & 0.238 & 0.027 & 2.106 \\
\hline & Q1 & Q3 & 0.229 & 0.025 & 2.096 \\
\hline & Q1 & Q4 & 0.433 & 0.040 & 4.742 \\
\hline & Q2 & Q3 & 0.964 & 0.361 & 2.569 \\
\hline & Q2 & $\mathrm{Q} 4$ & 1.820 & 0.442 & 7.498 \\
\hline & Q3 & Q4 & 1.888 & 0.433 & 8.233 \\
\hline \multirow[t]{6}{*}{ Q 2} & Q1 & Q2 & 0.213 & 0.050 & 0.905 \\
\hline & Q1 & Q3 & 0.218 & 0.050 & 0.955 \\
\hline & Q1 & Q4 & 0.129 & 0.027 & 0.620 \\
\hline & Q2 & Q3 & 1.026 & 0.331 & 3.175 \\
\hline & Q2 & Q4 & 0.606 & 0.173 & 2.120 \\
\hline & Q3 & $\mathrm{Q} 4$ & 0.590 & 0.176 & 1.978 \\
\hline \multirow[t]{6}{*}{ Q 3} & Q1 & Q2 & 0.065 & 0.003 & 1.305 \\
\hline & Q1 & Q3 & 0.015 & $<0.001$ & 0.277 \\
\hline & Q1 & Q4 & 0.021 & 0.001 & 0.363 \\
\hline & Q2 & Q3 & 0.235 & 0.054 & 1.016 \\
\hline & Q2 & $\mathrm{Q} 4$ & 0.324 & 0.076 & 1.380 \\
\hline & Q3 & Q4 & 1.379 & 0.416 & 4.569 \\
\hline \multirow[t]{6}{*}{ Q 4} & Q1 & Q2 & 0.365 & 0.014 & 9.368 \\
\hline & Q1 & Q3 & 0.095 & 0.006 & 1.425 \\
\hline & Q1 & Q4 & 0.051 & 0.005 & 0.539 \\
\hline & Q2 & Q3 & 0.261 & 0.015 & 4.618 \\
\hline & Q2 & $\mathrm{Q} 4$ & 0.139 & 0.011 & 1.806 \\
\hline & Q3 & $\mathrm{Q} 4$ & 0.535 & 0.090 & 3.183 \\
\hline
\end{tabular}

Of the comparisons between ration groups, only the contrast between ration 14 and rations 41 , 42, and 47 grouped together show some evidence of a difference, Table 6. A p-value of 0.0836 gives weak evidence that the odds of death ratios for ration 14 is greater than combination of rations group 414247. Similarly, the lower endpoint for the $95 \%$ confidence interval for the odds ratio is 0.9119 which is close to 1 . Both results offer weak evidence of a difference in odds of death ratios. According to the estimated odds ratio, the estimated odds of death are 2.0022 times larger for ration 14 than for the combined ration group 414247. 
Table 6: Exploratory analysis on contrasts for Ration by Slope interactions

\begin{tabular}{|l|l|l|l|l|l|l|}
\hline Label & Est & Std Err & P-value & Odds Ratio & Lower Odds Ratio & Upper Odds Ratio \\
\hline Ration_Group 14 vs. 414247 & 0.6943 & 0.4011 & 0.0836 & 2.0022 & 0.9119 & 4.3962 \\
\hline Slope SevW vs. Flat & 1.1096 & 0.4390 & 0.0116 & 3.0331 & 1.2823 & 7.1744 \\
\hline Slope SevW vs. ME & 1.2543 & 0.4673 & 0.0073 & 3.5055 & 1.4022 & 8.7637 \\
\hline Slope MNW vs. MW & -1.1055 & 0.6122 & 0.0711 & 0.3310 & 0.09965 & 1.0997 \\
\hline Slope MNW vs. Nor & -1.3753 & 0.7303 & 0.0598 & 0.2528 & 0.06036 & 1.0584 \\
\hline Slope SevW vs. MNW & 1.8286 & 0.6381 & 0.0042 & 6.2252 & 1.7813 & 21.7555 \\
\hline Slope MNW vs. Sou & -1.2171 & 0.6589 & 0.0648 & 0.2961 & 0.08133 & 1.0778 \\
\hline
\end{tabular}

The simple effects of slope are also analyzed using unadjusted odds ratios. When severe west slope is compared with flat, moderate east, and moderate northwest slopes, the odds of death ratios are all higher for the severe west slopes at the 95\% confidence level. Since each of these contrasts has a small p-value, these results present strong evidence of a difference. Finally, comparing moderate northwest slope to each of the moderate west, north, and south slopes, weak to moderate evidence indicates that the odds of death ratio are less for a moderate northwest slope.

In Table 7, SqFtPerHead quartiles are compared at a specific pen average weight PenDailyAvgWeight quartile. When PenDailyAvgWeight is in Q1 $(670 \mathrm{lbs})$, the odds of death ratio are higher for SqFtPerHead Q1 and $2\left(170\right.$ and $\left.190 \mathrm{ft}^{2}\right)$ than Q3 $\left(240 \mathrm{ft}^{2}\right)$ at the 95\% confidence level. Thus, lighter cattle in dense pens indicate a higher odds of death ratio than lighter cattle in spacious pens. Next, when PenDailyAvgWeight is in Q 2 (900 lbs), the odds of death ratios are higher for SqFtPerHead Q1 $\left(170 \mathrm{ft}^{2}\right)$ than Q3 and $4\left(240\right.$ and $\left.1350 \mathrm{ft}^{2}\right)$. Likewise, the odds of death ratio are higher for SqFtPerHead Q2 $\left(190 \mathrm{ft}^{2}\right)$ than Q3 $\left(240 \mathrm{ft}^{2}\right)$ given PenDailyAvgWeight is in Q2 $(900 \mathrm{lbs})$. Once more, lighter cattle have a higher odds of death ratio in dense pens than spacious pens. Hence, lighter cattle show a change in deaths according to pen density while heavier cattle do not.

Table 7: Exploratory analysis on contrasts for PenDailyAvgWeight quartiles by SqFtPerHead quartiles interactions

\begin{tabular}{|l|l|l|l|l|l|l|}
\hline Label & Est & Std Err & P-value & Odds Ratio & Lower Odds Ratio & Upper Odds Ratio \\
\hline $\begin{array}{l}\text { Avg Weight Q1 \& } \\
\text { Square Feet Q1 vs. Q3 }\end{array}$ & 2.6519 & 1.2799 & 0.0384 & 14.1808 & 1.1525 & 174.49 \\
\hline $\begin{array}{l}\text { Avg Weight Q1 \& } \\
\text { Square Feet Q2 vs. Q3 }\end{array}$ & 2.2899 & 1.1108 & 0.0394 & 9.8735 & 1.1179 & 87.2015 \\
\hline $\begin{array}{l}\text { Avg Weight Q2 \& } \\
\text { Square Feet Q1 vs. Q3 }\end{array}$ & 1.3512 & 0.5322 & 0.0112 & 3.8621 & 1.3601 & 10.9663 \\
\hline $\begin{array}{l}\text { Avg Weight Q2 \& } \\
\text { Square Feet Q1 vs. Q4 }\end{array}$ & 1.8865 & 0.9549 & 0.0483 & 6.5960 & 1.0139 & 42.9093 \\
\hline $\begin{array}{l}\text { Avg Weight Q2 \& } \\
\text { Square Feet Q2 vs. Q3 }\end{array}$ & 1.1010 & 0.5491 & 0.0451 & 3.0070 & 1.0245 & 8.826 \\
\hline
\end{tabular}

\section{Conclusion}

After obtaining the full data set, descriptive statistics summarized availability of information for certain variable levels. Some interactions did not have pens at certain levels and confounding was noticeable. Then, a statistical model was developed and odds ratio confidence intervals were computed for statistical inference. The Bonferroni adjusted odds ratios show low dry matter intake levels have significantly higher odds of death than other levels during July $16-18$. 
Likewise, declines in dry matter intake indicated higher odds of death than when dry matter intake levels were increasing during July $16-18$. Moreover, when pen area increases, the odds of death are greater for the heaviest pens of cattle than for the lightest pens of cattle. The exploratory data analysis showed the estimated odds of death are 2 times larger for the heifer finisher ration than for the combined Beta-agonist rations. The severe west slope shows evidence of higher odds of death than each of the following slopes: flat, moderate east, and moderate northwest. Finally, odds of death are higher for lighter cattle in dense pens than spacious pens.

\section{Summary}

This study investigates factors during a heat wave in addition to temperature, which could have an effect on summer feedlot deaths for a single location over a 2 week period in 2010 . Descriptive statistics were used to understand the structure of the feedlot data. A generalized linear mixed model was developed based on the significant factors. Additionally, exploratory analyses were applied to further understand the interactions between factor levels.

Factors contributing to cattle deaths, such as daily pen, cattle, and feed type traits, were recorded at a single Midwestern feed yard in July 2010. Weather data were obtained from the feed yard and a neighboring town about 4 miles away. Rather than using multiple weather variables, a single comprehensive climate index that summarizes several weather variables was used to capture the apparent feel of the weather.

Descriptive statistics were obtained to identify whether or not the data set contained pens for each level of the factors and two-way interactions, before constructing a statistical model. Interactions with missing pens were not included in the statistical model due to insufficient information. To improve the structure of the data, days were grouped into three periods: pre,during, and post-heatwave; pen slope was characterized as flat, moderate (east, northeast, northwest, west ), north , severe west, and south; and feed type traits were categorized based on type of ration and dietary feed additive.

A generalized linear mixed model was built using those variables and two-way interactions with adequate pen and death counts. The Bonferroni adjusted odds ratios from the model show pens with lower feed consumption are associated with more deaths. Dry matter intake per head and pen area $\left(\mathrm{ft}^{2} / \mathrm{head}\right)$ are also associated with cattle deaths. For dry matter intake, the odds of death are higher when intake is declining. Increasing pen space/animal decreased deaths in lighter weight groups but not in heavier cattle.

Exploratory data analysis uses unadjusted odds ratios to provide more insight into the data. Ration and slope are additional factors associated with feedlot deaths. Weak evidence indicates that the heifer finishing ration has larger odds of death than rations containing Beta-agonists. Hence, the Beta-agonists were not found to be associated with cattle deaths. However, the topology of the pen is an important factor. There is strong evidence a severe west slope is a factor contributing to summer feedlot deaths.

Because the feedlot data were not obtained by an experimental design, many difficulties arise. As indicated above, confounding is present within the data set. Thus, definitive hypotheses cannot be tested with these data. Nonetheless, relevant information can still be obtained. More deaths 
for rations containing Beta-agonists are needed in order for these rations to be analyzed individually. Further research at another location using these rations with abundant deaths would provide more information as to whether the above tendencies hold.

\section{References}

Mader, T. L. 2003. Environmental stress in confined beef cattle. Journal of Animal Science 81 E110.

Mader, T.L., M. S. Davis, and T. Brown-Brandl. 2006. Environmental factors influencing heat stress in feedlot cattle. Journal of Animal Science 84:3 712-719.

Mader, T. L. and J. B. Gaughan. 2009. Heat and cold stress effects on beef cattle. $18^{\text {th }}$ ADSA Discover conference on Food Animal Agriculture: Effect of the Thermal Environment on Nutrient and Management Requirements of Cattle. Brown County Inn, Nashville, IN.

Mader, T. L., L. J. Johnson, and J. B. Gaughan. 2010. A comprehensive index for assessing environmental stress in animals. Journal of Animal Science 88: 2153-2165.

Morel, J. G., M. C. Bokossa, and N. K. Neerchal. 2003. Small Sample Correction for the Variance of GEE Estimators. Biometrical Journal 45:4 395-409.

SAS Institute Inc. 2010. SAS/STAT® 9.22 User’s Guide. Cary, NC: SAS Institute Inc. 